\title{
The Tol 2 transposable element of the medaka fish: an active DNA-based element naturally occurring in a vertebrate genome
}

\author{
Akihiko Koga and Hiroshi Hori* \\ Division of Biological Sciences, Graduate School of Science, Nagoya University, \\ Nagoya 464-8602, J apan
}

\begin{abstract}
Several DNA-based transposable elements are known to be present in vertebrate genomes, but few of them have been demonstrated to be active. The Tol 2 element of the medaka fish is one such element and, therefore, is potentially useful for developing a gene tagging system and other molecular biological tools applicable to vertebrates. Towards this goal, analyses of the element at the molecular, cellular and population levels are in progress. Results so far obtained are described here.
\end{abstract}

Transposable elements as genetic tools. Transposable elements serve, in various organisms, as powerful tools for genetic analyses. The simplest form of application is as transformation vectors using an element carrying a gene or other DNA fragments (cf. Rubin and Spradling 1982). Inducing insertion mutations, followed by phenotype screening, is called gene tagging and allows quick cloning of causative genes (cf. Bingham et al. 1982, Searles et al. 1982). Similar usages are in promoter trapping and enhancer trapping, with an element that carries a marker gene lacking a promoter or containing a weak promoter (cf. O'Kane and Gehring 1987). These are applications common to both of the two major classes of transposable elements: RNA-mediated elements and DNA-based elements (class I and class II elements, respectively, of Finnegan 1992). A usage unique to the latter is to induce further changes such as reversion mutations due to precise excision or a range of mutations by imprecise excision (Salz et al. 1987). Another advantage of DNAbased elements is that transposition can be started, if the necessary strains have been prepared, merely by crossing the strains (cf. Cooley et al. 1988).

Such technology using DNA-based elements was established as early as in the 1970's in bacteria, and in the mid1980's in nematodes, Drosophila and other eukaryotic model organisms. In vertebrates, however, discovery of active elements is relatively recent and, for this reason, their potential has been less fully realized.

Discovery of transposable elements. New transposable elements are most frequently encountered as mutations occurring in genes of host organisms. There are

* Corresponding author. E-mail: hori@bio.nagoya-u.ac.jp many examples of mutant genes for which structural analysis resulted in identification of extra DNA fragments. Such insertion appears more likely to happen in cases of high mutation rates. The Ac element of maize and the $P$ element of Drosophila were first hypothesized to be present as genetic factors that cause high rates of mutant phenotypes of kernel color (McClintock 1948) and of fertility (Kidwell et al. 1979), respectively. P was actually identified and cloned as an insertion sequence in the Drosophila white locus (Rubinet al. 1982) and Ac in the maize Waxy locus (F edoroff et al. 1983).

Also in other organisms, new elements were discovered, one after another, and, at the beginning of the 1990's, DNA-based elements had become considered to be almost universal components of genomes. Although many RNAmediated elements had been reported, vertebrates were an exception in that DNA-based elements had only been found in the frog Xenopus Iaevis.

Search for transposable elements in vertebrates. With advances in technology and the accumulation of information, a new method for detecting transposable elements was devised based on similarity of nucleotide sequences. Elements having sequence similarities with the Drosophila mariner element and the nematode Tcl element werethen found in a wide range of organisms and called the mariner/Tc1 family or the Tc1/mariner family (Plasterk 1996, Hartl et al. 1997). In addition to the wide distribution, it is a feature of this family that the copy number in the genome is high, which is significant for finding elements by methods that rely on sequence similarity.

The initial attempt to search a database for a nucleotide sequence having similarity with mariner/Tcl family ele ments provided the first example, from the catfish (Heni- 
Table 1. Transposable elements discovered of demonstrated to be active in vertebrates

\begin{tabular}{|c|c|c|c|c|}
\hline Host & Element & $\begin{array}{l}\text { Evidence for } \\
\text { activity }\end{array}$ & $\begin{array}{l}\text { I solation of } \\
\text { autonomous copy }\end{array}$ & Reference \\
\hline frog & 1723 & No & No & Kay and Dawid (1983) \\
\hline frog & $\mathrm{Vi}$ & No & No & Schubiger et al. (1985) \\
\hline frog & Tx1 & No & No & Garrett and Carroll (1986) \\
\hline catfish & I pTc1 & No & No & Henikoff (1992) \\
\hline hagfish & Tes1 & No & No & Heierhorst et al. (1992) \\
\hline salmon & SALT1 & No & No & Goodier and Davidson (1994) \\
\hline zebrafish & Tbr1 & No & No & Radice et al. (1994) \\
\hline salmon & Tss1 & No & No & Radice et al. (1994) \\
\hline human & tcrbMLE & No & No & Morgan (1995) \\
\hline zebrafish & Tdr1 & No & No & Izsvák et al. (1995) \\
\hline medaka fish & Tol 1 & $\mathrm{No}^{\mathrm{a})}$ & No & Koga et al. (1995) \\
\hline human & humar1 & No & No & Oosumi et al. (1995) \\
\hline zebrafish & Tdr2 & No & No & I vics et al. (1996) \\
\hline salmon & Tss2 & No & No & I vics et al. (1996) \\
\hline human & Tigger 1 & No & No & Smit and Riggs (1996) \\
\hline human & MITE & No & No & Reiter et al. (1996) \\
\hline frog & $\mathrm{TXr}$ & No & No & Lam et al. (1996b) \\
\hline medaka fish & Tol2 & Yes & Yes & Koga et al. (1996) \\
\hline zebrafish & Tzf & Yes & No & Lam et al. (1996a) \\
\hline salmon & Sleeping Beauty & Yes & $Y e s^{b)}$ & I vics et al. (1997) \\
\hline nematode & TC3 & Yes & Yes & Raz et al. (1997) \\
\hline Drosophila & mariner & Yes & Yes & Fadool et al. (1998) \\
\hline zebrafish & Tdr2 & No & No & Gottgens et al. (1999) \\
\hline
\end{tabular}

a) Transposition activity has now been demonstrated (unpublished).

b) An autonomous copy was synthesized.

koff 1992). The method was subsequently expanded to include PCR (polymerase chain reaction) and many more elements were discovered (Table 1). However, those found in the early stage were all inactive elements, not carrying a complete internal gene.

Active elements found in medaka fish. The medaka fish Oryzias latipes is a freshwater teleost species native to Asia, including China, J apan and Korea. This fish serves as a major material for research in genetics and many body color mutants have been identified. Among mutants stocked at Nagoya University, three albino strains having different alleles at the i (color interferer) locus were established by H. Tomita (Yamamoto 1969, Tomita 1975). Tyrosinase is the key enzyme in melanin biosynthesis, and many cases of al binism are known to be caused by its defects (Shibahara et al. 1990, Yokoyama et al. 1990). We first cloned the wild-type tyrosinase gene of the medaka fish (Inagaki et al. 1994) and then examined the structures of the genes of the albino mutant strains (Fig. 1). The $i^{1}$ allele, which causes a complete al bino phenotype, was shown to carry an extra 1.9-kb DNA fragment in the coding region (Koga et al. 1995). The element has structural characteristics of a DNA-based transposable element, and we named it Tol 1 (Transposable el ement of Oryzias latipes, number 1). Similarly, the $\mathrm{i}^{4}$ allele, which causes a quasi-albino phenotype, was demonstrated to contain a $4.7-\mathrm{kb}$ insertion, and this element was named Tol2 (Koga et al. 1996). The third allele, $\mathrm{i}^{5}$, causing a weak albino phenotype, was shown to have a Tol 2 copy at a position different from that of the $i^{4}$ allele (unpublished).

Of the two elements, Tol 2 was demonstrated to be active soon after its discovery. We conducted PCR with primers encompassing the Tol 2 insertion point. As expected, the length of the major PCR product was $5.2 \mathrm{~kb}$, that is, the length between the primers $(0.5 \mathrm{~kb})$ plus the length of the Tol 2 copy $(4.7 \mathrm{~kb})$. In addition to this major product, a $0.5 \mathrm{~kb}$ fragment was observed. Cloning and sequencing analyses of this short fragment revealed footprints of excision of Tol2. This was the first report of an active DNA-based element in a vertebrate. The method applied was what has long been the major approach for many model organisms, that is, analysis of the structure of mutant genes. Mutations, especially those that have newly arisen, are certainly a pointer of active elements. Tomita's lifetime, patient work on collecting spontaneous mutations was the basis for our detection of active elements.

Structure of Tol2. Tol2 carries imperfect terminal inverted repeats of $17 \mathrm{bp}$ and $19 \mathrm{bp}$, and three subterminal repeats of about $30 \mathrm{bp}$ located proximal to the right terminal inverted repeat. It also contains internal inverted repeats of about $300 \mathrm{bp}$, which is unique among DNAbased el ements so far identified (Fig. 2). 


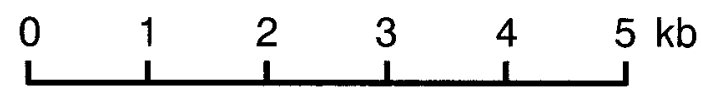

\section{Wild type}
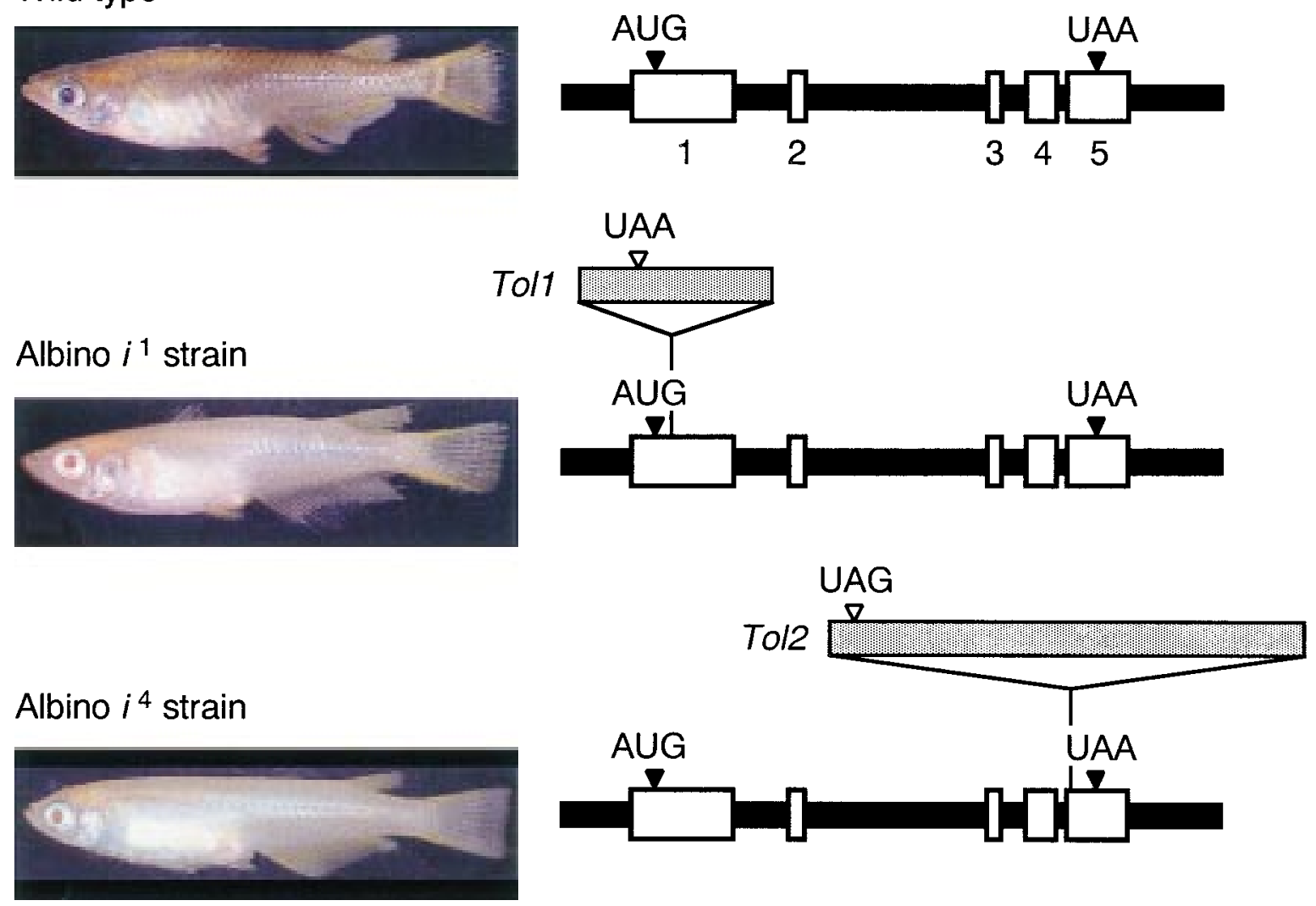

Fig. 1. Structures of medaka fish tyrosinasegenes. The tyrosinasegene consists of five exons (I nagaki et al. 1998) that produce a protein of 540 amino acids (Inagaki et al. 1994), as shown in the wild-type gene structure. The black arrowheads indicate the locations of the first methionine codon and the stop codon of the wild-type gene. The $\mathrm{i}^{1}$ albino strain exhibits a complete albino phenotype and its tyrosi nase gene contains a Tol 1 element in the first exon. The $\mathrm{i}^{4}$ al bino strain shows a quasi-al bino phenotype and its tyrosinase gene carries a Tol 2 el ement in the fifth exon. The open triangles indicate the locations of stop codons on the assumption that transcription continues into the transposable elements and translation proceeds al ong the transcripts.

The entire element is $4.7 \mathrm{~kb}$ in length and contains a gene, composed of four exons, for its transposase. The amino acid sequence of the gene has similarities, in some blocks, to the sequences of elements of the hAT family (Calvi et al. 1991, Atkinson et al. 1993), which includes hobo of Drosophila (McGinnis et al. 1983), Ac of maize ( $F$ doroff et al. 1983) and Tam3 of snapdragon (Sommer et al. 1985). The amino acid blocks are located in three segments previously reported to be conserved among the hAT family elements (Feldmar and Kunze 1991).

Transposase gene. Four open reading frames were detected in the Tol 2 copy first found in the tyrosinase gene, designated as Tol 2-tyr. These were expected to constitute a gene for a transposase of Tol2, as is the case for Ac and hobo, and this proved true. We isolated cDNAs for mRNAs that have sequence similarity with the open reading frames from medaka fish cells, and sequencing analysis revealed that the four open reading frames roughly correspond to exons, with two major forms of Tol2 mRNAs present in medaka fish cells (Koga et al. 1999). One form comprises all four exons and encodes a 685 -amino acid polypeptides, assuming that translation is initiated at the first encountered AUG codon. The other mRNA is composed of exons 2 to 4 and includes a frame for 453 amino acids. We synthesized the longer mRNA by in vitro transcription and injected it into fertilized eggs together with a donor plasmid and a target plasmid, resulting in transposition from the donor to the target plasmid (Fig. 3). We also conducted a control experiment in which the two plasmids were injected without the mRNA and, in this case, transposition was not observed. The materials we used were $\mathrm{O}$. latipes (medaka fish) and $\mathrm{O}$. melastigma (Indian medaka fish). Thelatter species does not contain Tol 2 in its genome. Therefore, our results clearly demonstrated that the gene carried by Tol 2 is for a transposase and the transposase has entire activity for cut-and-paste transposition (Koga and Hori 2000). 


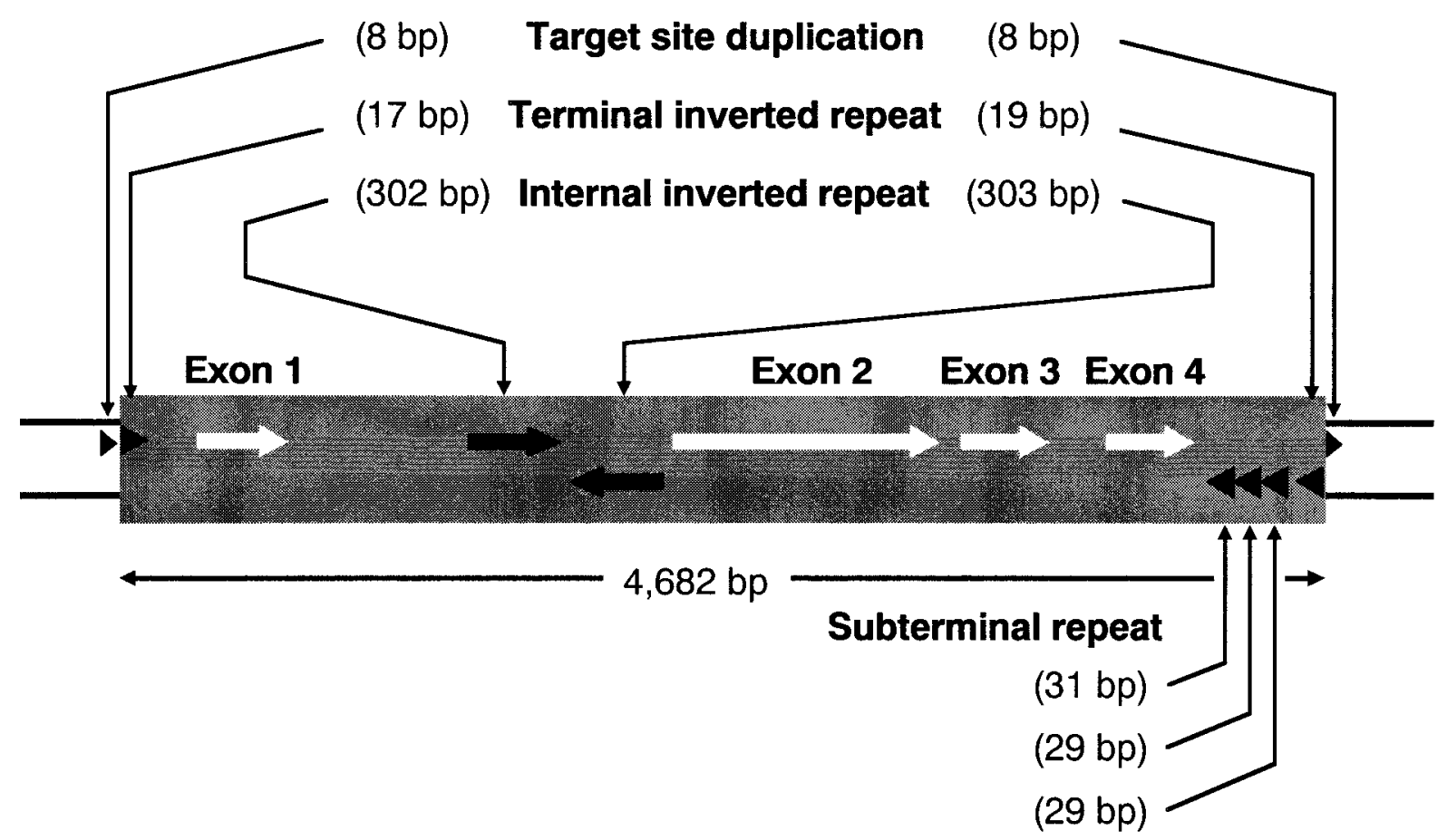

Fig. 2. Structure of the Tol 2 element. Tol 2 is 4,682 bp in length and its total sequence is available in DDBJ /EMBL/GenBank under accession number D84375. The black arrows and arrowheads indicate repeated sequences. The white arrows show exons for the transposase gene. Components are not drawn to scale. The internal inverted repeats constitute another, short transposable el ement called Angel, which is present in $10^{3}$ to $10^{4}$ copies in the genome of zebrafish (I zsvák et al. 1999) but not in the medaka fish genome except in Tol 2 (Koga et al. 2000).

Transposition activity was also shown in zebrafish for both excision (Kawakami and Shima 1999) and insertion (Kawakami et al. 2000).

Target site duplication. The method described above for detecting transposition from donor to target plasmids all owed us to collect many independent insertion products. We compared the sequences of target site duplications among 20 examples and determined the length of the duplication to be $8 \mathrm{bp}$ in all cases, with no apparent tendency to favor specific nucleotides. Wealso examined the flanking sequences of $20 \mathrm{Tol} 2$ copies present in the genome, and obtained the same results (unpublished results).

Copy number and variation. Genomic Southern blot analysis of ten species in the genus Oryzias demonstrated that Tol 2 is present in two, O. latipes (medaka fish) and O. curvinotus (Hainan medaka fish, inhabiting Southern China and Vietnam), with copy numbers, estimated by counting hybridization bands, of about 20 and about 30 , respectively. Linkage analysis of the copies showed them to be dispersed throughout the genome (Koga et al. 2000).

It is generally observed that DNA-based elements comprise both "full-length" copies and defective, shorter copies. The latter are mostly the products of internal deletion. However, this does not apply to Tol2. We examined the variation in the structures of more than 200 copies of Tol2, and showed that most, and possibly all, of the copies are $4.7 \mathrm{~kb}$ in length. There appeared to be no or very limited internal deletions, as shown by restriction mapping with six restriction sites. An unexpectedly small amount of variation was observed even at the nucleotide sequence level; that is, no sequence variation was evident among five randomly chosen copies. We inferred from these results that all or most of the Tol2 copies, including Tol2-tyr, are autonomous (Koga and Hori 1999).

Transposition in the germline. Tol 2 is not as active as Ac of maize or $\mathrm{P}$ of Drosophila, at least under usual laboratory conditions, because genomic Southern blots of fish of two consecutive generations have not shown any band unique to the second generation (Koga and Hori 1999, Koga et al. 2000). However, transposition activity in the germline is supported by the results of Southern blotting of random samples collected from natural populations. This analysis revealed that band patterns differ from one fish to another (Koga and Hori 1999). This result indicates that the "allele frequency" at the presence/absence "locus" of Tol2 is extremely low for all the Tol 2 copies and that these copies are continuously moving, or have recently moved in an explosive fashion, in the germline.

Transposition in the germline from an injected Tol2 clone has already been observed. We injected fertilized 
Donor

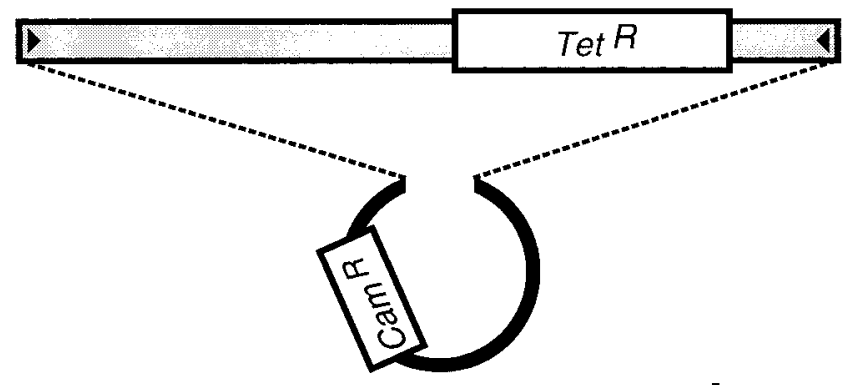

Target

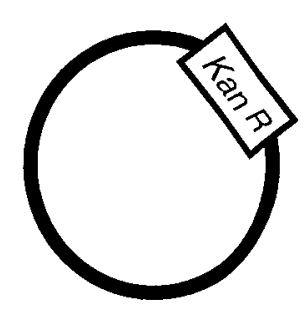

Inject into fertilized eggs.

Recover plasmids from embryos.

Introduce into bacteria.

Screen colonies with Kan and Tet.

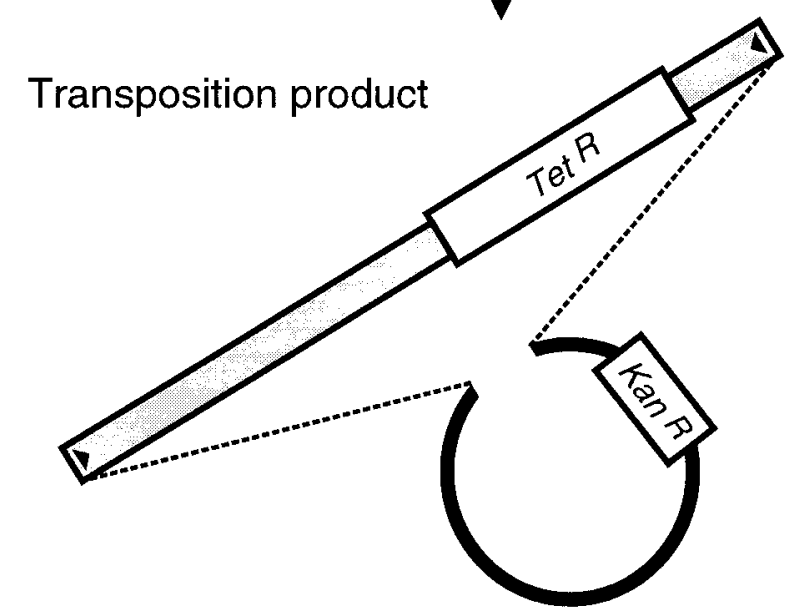

Fig. 3. Protocols for experiments to detect transposition of Tol2. The donor and target plasmids were microinjected into fertilized eggs with or without mRNA. Screening after these procedures resulted in detection of many independent transposition products.

eggs of O. melastigma, in which Tol 2 is absent, with a plasmid clone of Tol 2 and the transposase mRNA. The eggs were raised to adults and two of the 13 fish examined were shown to be positive after tail biopsy and PCR for Tol2. One of the two fish was crossed to another and five of their 46 offspring were shown to contain Tol 2 in their genomes. Cloning and sequencing of Tol 2 and its flanking chromosomal regions revealed a precise Tol 2 insertion carrying an 8-bp target site duplication (unpublished results). It is thus evident that transposition of Tol2 occurred in the germline of the first generation. Similar results were obtained for the zebrafish (Kawakami et al. 2000) that also lacks Tol 2 in its genome, except for the internal inverted repeat regions.

Development of genetic tools. Tol 2 is one of the few DNA-based elements so far demonstrated to be active in vertebrates. We have therefore concentrated attention on this element with a view to development of genetic tools.

Research for the same purpose is being conducted by several groups with different elements, and the most successful element in this respect appears to be Sleping Beauty (I vics et al. 1997, I zsvák et al. 2000). Autonomous copies of mariner/Tcl elements have yet to be found in vertebrates, but these researchers have made an autonomous copy by artificially recombining nonautonomous copies from eight fish species. This element is active not only in fish but also in various other vertebrate genomes including the human genome. Other promising elements are the exogenous elements Tc3 and mariner from the nematode and Drosophila, respectively, for which mobility in zebrafish has al ready been demonstrated (Raz et al. 1997, Fadool et al. 1998).

The elements described above are all members of the mariner/Tcl family. Tol2, in contrast, belongs to the hAT family, elements of which are also widely distributed among organisms (cf. Koga et al. 1999). hAT family ele- 
ments have features different from those of the mariner/ Tcl family. One difference is the element size, mariner/ Tcl el ements being mostly 1 to $2 \mathrm{~kb}$ and autonomous hAT elements typically 3 to $6 \mathrm{~kb}$. The larger size of the latter might be an advantage for carrying large DNA fragments. Another advantage might be expected concerning the distance between the "cut" and the "paste" sites. Studies of this distance with the maize Ac element showed that it transposes preferentially to nearby regions (Machida et al. 1997). If this is also the case for Tol2, it may be useful for local targeting, creating chromosomal deletions and inversions, and generating large numbers of mutations within a single gene.

The Tol 1 element of the medaka fish also has potential regarding development of molecular biological techniques because its transposition activity has recently been shown. Spontaneous reversion mutation of the tyrosinase gene was observed in the $\mathrm{i}^{1}$ al bino strain, and PCR and sequencing analyses revealed excision footprints of the element (unpublished results). In addition, the particular Tol 1 insertion that generated the $\mathrm{i}^{1}$ allele has been shown to be a recent event (Koga and Hori 1997). It is not known which other element Tol 1 might be related to, because an open reading frame has not been found in any of the Toll copies we have so far examined. The only inference we can make at present is that Tol 1 is not a mariner $/ \mathrm{Ccl}$ element because its target site duplication is not the "TA" dinucleotide but rather 8-bp heterogeneous nucleotides. Efforts are now being made to identify an autonomous Tol 1 copy.

Evolution of Tol2. Apart from the molecular mechanisms of transposition and the application to biotechnology, Tol 2 has an interesting feature with regard to its evolution. Horizontal transfer is thought to be a significant factor for survival of transposable elements (Kidwell 1992, Lohe et al. 1995). Evidence of its presence is typically provided by observation of inconsistency between the phylogeny of the element and that of its host species. However, among many examples, only a few allow dear conclusions because in many cases explanations other than horizontal transfer cannot be excluded (cf. Capy et al. 1994). Tol 2 was found to be highly homogeneous at the nucleotide sequence level among several samples from collection sites representative of the entire distribution area of $\mathrm{O}$. latipes. High sequence homogeneity was shown even between the two Tol2-carrying species, $\mathrm{O}$. latipes and $\mathrm{O}$. curvinotus. Comparing this low sequence variation in Tol 2 with that of a nuclear gene of the host species, we proposed horizontal transfer of Tol 2 from one of the two species to the other or into both of them from an unknown common source (Koga et al. 2000). If our inference is true, the mechanism by which the element crossed the species barrier is an interesting theme from the viewpoint of genome evolution, because horizontal transfer of transposable elements raises the possibility of horizontal transfer of host factors carried by transposable el ements.

The authors owe much to Professor Hideo Tomita of Nagoya University, who passed away in 1998, for his support of our work on medaka fish transposable elements, including Tol2. Thestaff of the World Medaka Aquarium of Nagoya City Higashiyama Zoological Garden provided us with samples of various species of fish and this was of great assistance in conducting evolutionary studies.

\section{REFERENCES}

Atkinson, P. W., Warren, W. D., and O'Brochta, D. A. (1993) The hobo transposable element of Drosophila can be cross-mobilized in houseflies and excises like the Ac element of maize. Proc. Natl. Acad. Sci. USA 90, 9693-9697.

Bingham, P. M., Kidwell, M. G., and Rubin, G. M. (1982) The molecular basis of P-M hybrid dysgenesis: the role of the $P$ element, a P-strain-specific transposon family. Cell 29, 9951004.

Calvi, B. R., Hong, T. J ., Findley, S. D., and Gelbert, W. M. (1991) Evidence for a common evolutionary origin of inverted repeat transposons in Drosophila and plants: hobo, Activator, and Tam3. Cell 66, 465-471.

Capy, P., Anxolabéhère, D., and Langin, T. (1994) The strange phylogenies of transposable elements: are horizontal transfers the only explantation? Trends Genet. 10, 7-12.

Cooley, L., Kelley, R., and Spradling, A. (1988) Insertional mutagenesis of the Drosophila genome with single $P$ elements. Science 239, 1121-1128.

Fadool, J . M., Hartl, D. L., and Dowling, J . E. (1998) Transposition of the mariner element from Drosophila mauritiana in zebrafish. Proc. Natl. Acad. Sci. USA 95, 5182-5186.

Fedoroff, N., Wessler, S., and Shure, M. (1983) I solation of the transposable maize controlling el ements Ac and Ds. Cell 35, 235-242.

Feldmar, S, and Kunze, R. (1991) The ORF a protein, the putative transposase of maize transposable element Ac, has a basic DNA binding domain. EMBO J . 10, 4003-4010.

Finnegan, D. J. (1992) Transposable elements. Curr. Opin. Genet. Dev. 2, 861-867.

Garrett, J . E., and Carroll, D. (1986) Tx1: a transposable element from Xenopus laevis with some unusual properties. Mol. Cell. Biol. 6, 933-941.

Goodier, J . L., and Davidson, W. S. (1994) Tcl transposon-like sequences are widely distributed in Salmonids. J . Mol. Biol. 241, 26-34.

Gottgens, B., Barton, L. M., Grafham, D., Vaudin, M., and Green, A. R. (1999) Tdr2, a new zebrafish transposon of the Tc1 family. Gene 239, 373-379.

Hartl, D. L., Lohe, A. R., and Lozovskaya, E. R. (1997) Modern thoughts on an ancyent marinere: function, evolution, regulation. Annu. Rev. Genet. 31, 337-358.

Heierhorst, J ., Lederis, K., and Richter, D. (1992) Presence of a member of the Tcl-like transposon family from nematodes and Drosophila within the vasotocin gene of a primitive vertebrate, the Pacific hagfish Eptatretus stouti. Proc. Natl. Acad. Sci. USA 89, 6798-6802.

Henikoff, S. (1992) Detection of Caenorhabditis transposon homologs in diverse organisms. New Biol. 4, 382-388.

Inagaki, H., Bessho, Y., Koga, A., and Hori, H. (1994) Expression of the tyrosinase-encoding gene in a colorless melanophore mutant of the medaka fish, Oryzias latipes. Gene 150, 319324.

Inagaki, H., Koga, A., Bessho, Y., and Hori, H. (1998) The tyrosi- 
nase gene from medakafish: transgenic expression rescues albino mutation. Pigment Cell Res. 11, 283-290.

I vics, Z., Hackett, P. B., Plasterk, R. H., and Izsvák, Z. (1997) Molecular reconstruction of Sleeping Beauty, a Tcl-like transposon from fish, and its transposition in human cells. Cell 91, 501-510.

I vics, Z., I zsvák, Z., Minter, A., and Hackett, P. B. (1996) I dentification of functional domains and evolution of Tcl-like transposable elements. Proc. Natl. Acad. Sci. USA 93, 5008-5013.

I zsvák, Z., I vics, Z., and Hackett, P. B. (1995) Characterization of a Tcl-like transposable element in zebrafish (Danio rerio). Mol. Gen. Genet. 247, 312-322.

I zsvák, Z., I vics, Z., and Plasterk, R. H. (2000) Sleeping Beauty, a wide host-range transposon vector for genetic transformation in vertebrates. J. Mol. Biol. 302, 93-102.

Izsvák, Z., Ivics, Z., Shimoda, N., Mohn, D., Okamoto, H., and Hackett, P. B. (1999) Short inverted-repeat transposable elements in teleost fish and implications for a mechanism of their amplification. J. Mol. Evol. 48, 13-21.

Kawakami, K. and Shima, A. (1999) Identification of the Tol2 transposase of the medaka fish Oryzias latipes that catalyzes excision of a nonautonomous Tol 2 el ement in zebrafish Danio rerio. Gene 240, 239-244.

Kawakami, K., Shima, A., and Kawakami, N. (2000) Identification of a functional transposase of the Tol 2 element, an Ac-like element from the J apanese medaka fish, and its transposition in the zebrafish germ lineage. Proc. Natl. Acad. Sci. USA 97, 11403-11408.

Kay, B. K., and Dawid, I. B. (1983) The 1723 element: a long, homogeneous, highly repeated DNA unit interspersed in the genome of Xenopus laevis. J . Mol. Biol. 170, 583-596.

Kidwell, M. G., Kidwell J . F., and Sved, J . A. (1979) Hybrid dysgenesis in Drosophila melanogaster: a syndrome of aberrant traits including mutation, sterility, and male recombination. Genetics 86, 813-833.

Kidwell, M. G. (1992) Horizontal transfer. Curr. Opin. Genet. Dev. 2, 868-873.

Koga, A., and Hori, H. (1997) Albinism due to transposable element insertion in fish. Pigment Cell Res. 10, 377-381.

Koga, A., and Hori, H. (1999) Homogeneity in the structure of the medaka fish transposable element Tol2. Genet. Res. Camb. 73, 7-14.

Koga, A., and Hori, H. (2000) Detection of denovo insertion of the medaka fish transposable element Tol2. Genetics 156, 12431247.

Koga, A., Inagaki, H., Bessho, Y., and Hori, H. (1995) Insertion of a novel transposable element in the tyrosinase gene is responsible for an albino mutation in the medaka fish, Oryzias latipes. Mol. Gen. Genet. 249, 400-405.

Koga, A., Shimada, A., Shima, A., Sakaizumi, M., Tachida, H., and Hori, H. (2000) Evidence for recent invasion of the medaka fish genome by the Tol 2 transposable element. Genetics 155, 273-281.

Koga, A., Suzuki, M., Inagaki, H., Bessho, Y., and Hori, H. (1996) Transposable element in fish. Nature 383, 30.

Koga, A., Suzuki, M., Maruyama, Y., Tsutsumi, M., and Hori, H. (1999) Amino acid sequence of a putative transposase protein of the medaka fish transposable element Tol 2 deduced from mRNA nucleotide sequences. FEBS Lett. 461, 295-298.

Lam, W. L., Lee, T. S., and Gilbert, W. (1996a) Active transposition in zebrafish. Proc. Natl. Acad. Sci. USA 93, 10870-10875.

Lam, W. L., Seo, P., Robison, K., Virk, S., and Gilbert, W. (1996b) Discovery of amphibian Tcl-like transposon families. J . Mol. Biol. 257, 359-366.

Lohe, A. R., Moriyama, E. N., Lidholm, D. A., and Hartl, D. L. (1995) Horizontal transmission, vertical inactivation, and sto- chastic loss of mariner-liketransposable elements. Mol. Biol. Evol. 12, 62-72.

McClintock, B. (1948) Mutable loci in maize. Carnegie Inst. Wash. Year Book 47, 155-169.

McGinnis, W., Shermoen, A. W., and Beckendorf, S. K. (1983) A transposable element inserted just 5 ' to a Drosophila glue protein gene alters gene expression and chromatin structure. Cell 34, 75-84.

Machida, C., Onouchi, H., Koizumi, J ., Hamada, S., Semiarti, E., Torikai, S., and Machida, Y. (1997) Characterization of the transposition pattern of the Ac element in Arabidopsis thaliana using endonuclease I-Scel. Proc. Natl. Acad. Sci. USA 94, 8675-8680.

Morgan, G. T. (1995) Identification in the human genome of mobile elements spread by DNA-mediated transposition. J .Mol. Biol. 254, 1-5.

O'Kane, C. J ., and Gehring, W. J . (1987) Detection in situ of genomic regulatory elements in Drosophila. Proc. Natl. Acad. Sci. USA 84, 9123-9127.

Oosumi, T., Belknap, W. R., and Garlick, B. (1995) Mariner transposons in humans. Nature $\mathbf{3 7 8}, 672$.

Plasterk, R. H. A. (1996) The Tcl/mariner transposon family. In: Transposable Elements. Saedler, H., and Gierl, A. eds. Springer, Berlin, pp. 125-143.

Radice, A. D., Bugaj, B., Fitch, D. H., and Emmons, S. W. (1994) Widespread occurrence of the Tcl transposon family: Tcl-like transposons from teleost fish. Mol. Gen. Genet. 244, 606-612.

Raz, E., van Luenen, H. G. A. M., Schaerringer, B., Plasterk, R. H. A., and Driever, W. (1997) Transposition of the nematode Caenorhabditis elegans Tc3 element in the zebrafish Danio rerio. Curr. Biol. 8, 82-88.

Reiter, L. T., Murakami, T., Koeuth, T., Pentao, L., Muzny, D. M., Gibbs, R. A., and Lupski, J . R. (1996) A recombination hotspot responsible for two inherited peripheral neuropathies is located near a mariner transposon-like element. Nat. Genet. 12, 288-297.

Rubin, G. M., and Spradling, A. C. (1982) Genetic transformation of Drosophila with transposable element vectors. Science 218, 348-353.

Rubin, G. M., Kidwell, M. G., and Bingham, P. M. (1982) The molecular basis of P-M hybrid dysgenesis: the nature of induced mutations. Cell 29, 987-994.

Salz, H. K., Cline, T. W., and Schedl, P. (1987) F unctional changes associated with structural alterations induced by mobilization of a $\mathrm{P}$ element inserted in the Sex-lethal gene of Drosophila. Genetics 117, 221-231.

Schubiger, J . L., Germond, J . E., ten Heggeler, B., and Wahli, W. (1985) The Vi element. A transposon-like repeated DNA sequence interspersed in the vitellogenin locus of Xenopus laevis. J . Mol. Biol. 186, 491-503.

Searles, L. L., J okerst, R. S., Bingham, P. M., Voelker, R. A., and Greenleaf, A. L. (1982) M ol ecular cl oning of sequences from a Drosophila RNA polymerasell locus by $\mathrm{P}$ el ement transposon tagging. Cell 31, 585-592.

Shibahara, S., Okinaga, S., Tomita, Y., Takeda, A., Yamamoto, H., Sato, M., and Takeuchi, T. (1990) A point mutation in the tyrosinase gene of BALB/C albino mouse causing the cysteine - serine substitution at position 85. Eur. J. Biochem. 189, 455-461.

Smit, A. F., and Riggs, A. D. (1996) Tiggers and other DNA transposon fossils in the human genome. Proc. Natl. Acad. Sci. USA 93, 1443-1448.

Sommer, H., Carpenter, R., Harrison, R. J ., and Saedler, H. (1985) The transposable element Tam3 of Antirrhinum majus generates a novel type of sequence alteration upon excision. 
Mol. Gen. Genet. 199, 225-231.

Tomita, H. (1975) Mutant genes in the medaka. In: Medaka (Killifish): Biology and Strains, T. Yamamoto, ed. Yugakusha Publ., Tokyo, pp. 251-272.

Yamamoto, T. (1969) Inheritance of albinism in the medaka, Oryzias latipes, with special reference to gene interaction.
Genetics 62, 797-809.

Yokoyama, T., Silversides, D. W., Waymire, K. G., Kwon, B. S., Takeuchi, T., and Overbeek, P. A. (1990) Conserved cysteine to serine mutation in tyrosinase is responsible for the classical albino mutation in laboratory mice. Nucleic Acids Res. 18, 7293-7298. 